\title{
Insufficient Activity of MAPK Pathway Is a Key Monitor of Kidney-Yang Deficiency Syndrome
}

\author{
Wei Jun Ding, Ph.D., Shi Lin Yan, M.D., Ying Zi Zeng, M.S., ${ }^{1}$ Wei Hong Li, Ph.D.,', Azure Duan, Ph.D., \\ Tian E. Zheng, Ph.D., ${ }^{1}$ Min Liu, M.S., ${ }^{2}$ Cong E. Tan, M.D., ${ }^{1}$ Xiaokun Teng, Ph.D., ${ }^{3}$ and Jennifer Wan, Ph.D. ${ }^{4}$
}

\begin{abstract}
Objective: To explore the genetic characteristics and molecular regulator of Kidney-Yang Deficiency Syndrome (KDS).

Design: A typical KDS family was collected using a questionnaire of cold feeling and a 40-item scoring table of KDS based on Traditional Chinese Medicine (TCM), by single-blind method repeated annually over three years. Their transcriptomes were assayed by microarray and validated by RT-PCR and ELISA. Simultaneously, 10 healthy volunteers were recruited as controls and the same protocols were performed.

Results: This typical KDS family has 35 members, of whom 11 were evaluated as having severe KDS and 6 as having common KDS. Results of the cDNA microarray revealed that there were 420 genes/expressed sequence tags differentially expressed in KDS transcriptomes, indicating a global functional impairment in the mass-energyinformation carrying network of KDS patients, involving energy metabolism, signal transduction, development, cell cycle, and immunity. Pathway analysis by gene set enrichment assay (GSEA) and other tools demonstrated that mitogenic activated protein kinase (MAPK) is among the most insufficiently activated pathways, while the oxidative phosphorylation and glycolysis/gluconeogenesis pathways, the two main pathways relevant to ATP synthesis, were among the most excessively activated pathways in KDS patients. Results of RT-PCR and ELISA confirmed the status of insufficient activity of the MAPK pathway.

Conclusion: KDS patients undergo overall attenuated functions in the mass-energy-information carrying network. The marked low level of energy output in KDS may be primarily attributed to the insufficient activity of the MAPK pathway, which may be a key monitor for the abnormal energy metabolism and other impaired activities in KDS.
\end{abstract}

\section{Introduction}

K IDNEY YANG DEFICIENCY SYNDROME (KDS) is one of the primary concepts in Traditional Chinese Medicine (TCM). Although it affects millions of people, little research has been done to understand it. There are relatively few studies of the diversity of body temperature homeostasis, or the relationship between morphology and thermoregulation patterns. ${ }^{1-3}$ The definition of KDS is still rather vague in terms of conventional Western medicine, let alone its genetic background. However, we now have the tools to scan the global features of TCM syndromes with the extensive application of high throughput and genome scale tools such as microarray. ${ }^{4,5}$ TCM by its sheer nature is based on the analysis of various syndromes, and its holistic features and diagnosis methods can be verified by well-described protocols. ${ }^{5-7}$ Therefore, we believe that microarray may be an adequate tool to explore TCM syndromes such as KDS.

Some microarray experiments and relevant analysis designed to discover the basic features of KDS have been reported. Pan and colleagues evaluated a warming and tonic herb treatment for sibling patients from a KDS family with both clinical and molecular approaches to probe a clinical assessment for therapeutic efficacy of TCM. ${ }^{6}$ Wang and colleagues studied KDS and cold syndrome through family investigation and suggested that the formation and development of these two syndromes have not only genetic but also environmental factors. ${ }^{8} \mathrm{Wu}$ and colleagues established a discriminative mathematical model for the diagnosis of deficiency-cold syndrome, a major subtype of KDS, based on a

\footnotetext{
${ }^{1}$ Chengdu University of Traditional Chinese Medicine, Chengdu, China.

${ }^{2}$ Sichuan University, Sichuan, China.

${ }^{3}$ Shanghai Jiaotong University, Shanghai, China.

${ }^{4}$ Hong Kong University, Hong Kong, China.
} 
support vector machine (SVM), and demonstrated that the differentially expressed genes can be used to identify KDS. ${ }^{9}$ Yang et al. analyzed the KDS transcriptome module and indicated that KDS might be caused by physiologic imbalance and/or disorder of metabolite processes. ${ }^{10}$ However, we still have not discovered the key monitor or critical molecular pathway that can control the extensive disorders of energy and relative mass metabolism in KDS. Such studies will be important to understand the molecular regulators of KDS. In this paper we analyze a typical KDS family and attempt to assay the differentially expressed genes beyond microarray to uncover the molecular monitor of KDS patients.

\section{Materials and Methods}

\section{Epidemiological study and primary recruitment of KDS subjects}

We first performed a mid-scale epidemiological survey in order to identify research subjects who suffered from KDS in Chengdu, China. Because there is no Western medical definition of KDS, we chose the potential subjects based on a 10-question interview (Table 1). This questionnaire consists of the most frequent complaints of those suffering from unusual coldness, ${ }^{2}$ the major symptom of KDS. Subjects were chosen from those who answered "yes" to at least seven of the ten questions in the interview.

\section{Scoring table and quantitative criteria}

Potential subjects were diagnosed for KDS by five independent TCM practitioners according to a specific criteria system (GB/T15657-1995) promulgated by the Health Department of China. ${ }^{6,11}$ It includes the main results of TCM diagnosing techniques-interrogation, observation, auscultation, olfaction, and pulse palpation-with clinical manifestations attributed to KDS, such as dislike of cold and favor of warmth, whitish facial complexion, cold extremities, preference for staying in bed, tastelessness, clear and thin saliva, clear and copious urine, pale tongue with white coating, and slow and/or powerless pulse. ${ }^{6}$ This qualitative evaluation system was then transformed into a 40-item scoring table to quantify the various states of severity of KDS. A four-point system, 0 to 3, was proposed to grade

\section{Table 1. Questionnaire for the Cold Constitution}

1. Do you dislike cold drink and favor hot drink?

2. Do you often have pain in the fingertips or toes due to cold?

3. Do you need thicker clothes than others do?

4. Do you feel colder in a cold environment than others do?

5. Are you sensitive to a decrease of environmental temperature?

6. Do you prefer crouching in bed in winter?

7. Do you dislike being barefoot due to coldness?

8. Do you often wear socks or need an electric blanket for better sleep in winter?

9. Do you feel cold in an air-conditioned room when most people feel thermally comfortable?

10. Do you often wake up due to cold extremities or cold back?

The criteria for Kidney-Yang Deficiency Syndrome was 7 or more replies of "yes." clinical presentations as: none, light, medium, or heavy symptoms. The four main symptoms of KDS are: cold pain of lumbar and knee; profuse urination at night; long voiding of clear urine; and insufficient cubit pulse. ${ }^{12}$ Subjects were classified based on their total scores: $\leq 5$ points, healthy individual; 6-11 points, cold constitution; $12-18$ points, common KDS; and $\geq 19$ points, severe KDS. Such a standard is consistent with the clinical practice of treating KDS. People with common KDS and severe KDS were chosen for further tests.

\section{Isolation of total RNA}

As described previously, $10 \mathrm{~mL}$ samples of blood were collected from each subject. ${ }^{6}$ Total RNA was extracted using TRIzol reagent (Invitrogen Life Technologies, Carlsbad, CA) and the recommended protocol. All RNA samples were quantified by measuring the absorbance of $A_{260}$ and $A_{280} \mathrm{~nm}$ and followed by gel electrophoresis to ascertain the integrity of total RNA. The purified RNA was then stored in RNAsecure (Ambion, Austin, TX) at $-80^{\circ} \mathrm{C}$ prior to use.

\section{Microarray experiments}

Microarray assays were performed with Qiagen chips with 21,400 human 70mer Oligo DNA immobilized by the National Engineering Research Center for Beijing Biochip Technology, China, according to the protocols set by the ScanArray 5000 apparatus, (Packard BioScience) and analyzed using ImaGene3.0 software, (BioDiscovery, Inc.).

We designed three kinds of microchips to reciprocally confirm and effectively identify the key genes that may be attributed to KDS. The first chip was to hybridize both the transcriptomes of patients with severe KDS and healthy people, using the latter as the control group. The second was to compare patients with common KDS with the controls. The third chip was to compare a patient with severe KDS with her healthy husband, in order to filter out artifacts coming from differences in age, environment, and nutritional conditions. We conducted all hybridizations twice, with the interchange of both fluorescence Cy5 and Cy3 to decrease experimental error. Genes were identified as differentially expressed if the ratios were $\geq 2.0$ or $\leq 0.5$. To minimize artifacts arising from low expression values, only genes with raw intensity values for both Cy3 and Cy5 of $>2000$ counts were chosen for further analysis. $^{13}$

To functionally annotate the abnormally expressed genes, we retrieved and integrated information from these public databases: Kyoto Encyclopedia of genes and genomes (www .genome.jp/dbget-bin/); UniGene (www.ncbi.nlm.nih.gov/ UniGene/) and Gene Ontology (www.godatabase.org/dev/ database/archive). At first we linked the differentially expressed genes to the corresponding UniGene clusters. We then retrieved these databases of the biochemical pathways involved in the over- or under-expressed genes. Finally, disorders or diseases were summed and analyzed by means of gene set enrichment assay (GSEA) and other tools.

\section{Validation of the differentially expressed genes by $R T-P C R$}

Semiquantitative RT-PCR was performed with sequencespecific primers to validate the results observed in the microarray experiments. The primer pair for $\mathrm{MEK}_{1}$ was 
forward 5'-CGCTGTAGAACGCACCATAGAA-3' and backward 5'-TGGGAGAACTGAAGGA T GACGA-3'. The primer pair for extracellular signal-regulated kinase $(E R K)_{2}$ was forward 5'-AGGCGAATGCCAGCGTTAC-3' and backward 5'-GCGAAATAGATCCCTTTACCA-3'. The PCR productions were displayed by $1.8 \%$ gel electrophoresis and observed in the Gel-Doc system (Bio-Rad, Hercules, CA) and the data were analyzed by the attached software.

\section{Assay activity of two representative members of MAPK pathway by ELISA}

Two ELISA kits, MEK $_{1}$ (pSer218/222) (CBA030) and ERK $_{1 / 2}$ (Thr202/Tyr204) (CBA032) (Calbiochem, San Diego, $\mathrm{CA}$ ) were used. Following the manufacturer's protocols, we assayed the activities of two important MAPK members of every sample. The relative concentration of phosphorized amino acid in specific sites of certain enzymes represent its relative activity. Statistical differences were measured by $t$ test.

\section{Results}

\section{Questionnaire and TCM diagnosis}

Using the questionnaire mentioned above, we obtained 82 potential research subjects, 22 of whom belong to two families. One of the KDS pedigrees live in Xingxin town of Peng Zhou City, Sichuan Province, China, and have been intensively studied. ${ }^{6,8-10}$ Another family, which has 17 KDS patients and lives around Chengdu, Sichuan, China, was chosen as the subject for this present study (Fig. 1). Simultaneously, we recruited 10 healthy volunteers as control subjects. The control subjects' economic condition, living environment, age, and gender ratio matched the members of the KDS family.

\section{Reliability of diagnosis results}

In order to improve the reliability of TCM diagnosis, we invited 5 experts in TCM differentiation to independently evaluate all subjects in this family. Almost all of the results recorded by the 5 physicians were consistent (Fig. 2). The third physician may be stricter than the first and fifth physician, but there was no statistically significant difference among them. As the result, 11 individuals dispersed in generations II, III, and IV were evaluated as having severe KDS; 6 as having common KDS; and 6 as having cold constitution or as healthy individuals. The rest of the subjects were not evaluated due to death, nonavailability, or being too young.

We repeated the diagnosis on the same participants once a year for three years. Diagnosis in all three years was conducted on the first Saturday of December. Results revealed that every family member can be classified into the same KDS subtype in each year. For instance, in 2005 the average score of severe KDS within the pedigree was as high as 40.63 points, which was markedly high than that of the common KDS (15.38 points), cold constitution (6.89 points), or control group (3.24 points). Furthermore, the scores of every subtype of the KDS family were rather stable from 2003 through 2005, indicating the reliability of the subjects involved and the validity of the employed TCM criteria.

\section{Abnormally expressed genes related to energy metabolism as the major contributors in KDS transcriptome}

Results of the cDNA microarray experiments revealed that there were 420 differentially expressed genes/expressed sequence tags (EST) between KDS patients and the control group. Fully one third of these differentially expressed genes are related to energy metabolism. The remaining differentially expressed genes are associated with signal transduction, transport, development, cell cycle, and response to stress (Fig. 3). Concomitantly with the abnormally low level of energy output observed in KDS subjects, many other major functions such as signal transduction, mass transport, development, reproduction, metabolism, and immunity were found to be disturbed. In short, the results of our bioinformation analysis suggest the disturbance of the KDS transcriptome affects the mass-energy-information carrying network.

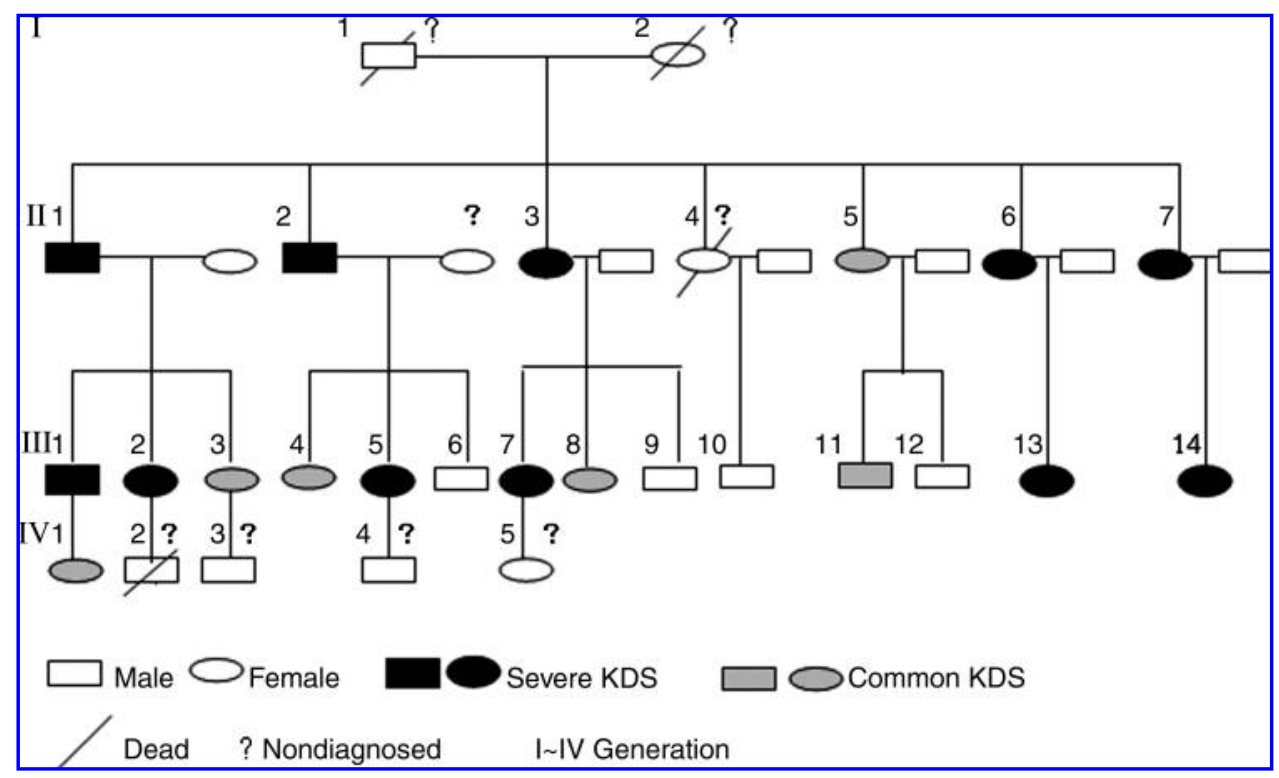

FIG. 1. Pedigree tree of a Kidney-Yang Deficiency Syndrome (KDS) family consisting of four generations and 35 members. The KDS subjects were evaluated by a questionnaire and subsequently by a 40 -item scoring table. Darker color indicates severe KDS, lighter color common KDS. A question mark indicates nondiagnosis due to death, nonavailability, or babies who were too young to be easily checked. 
FIG. 2. Traditional Chinese Medicine differentiation results of participants from a Kidney-Yang Deficiency Syndrome (KDS) pedigree (A). Five physicians marked every family number by the single blind method (B). Such diagnosis were performed three times from 2003 to 2005, on the first Saturday of December. Statistical results are presented as mean \pm standard deviation. sKDS, severe KDS, KDSc, KDS constitution, Contrl, healthy control.

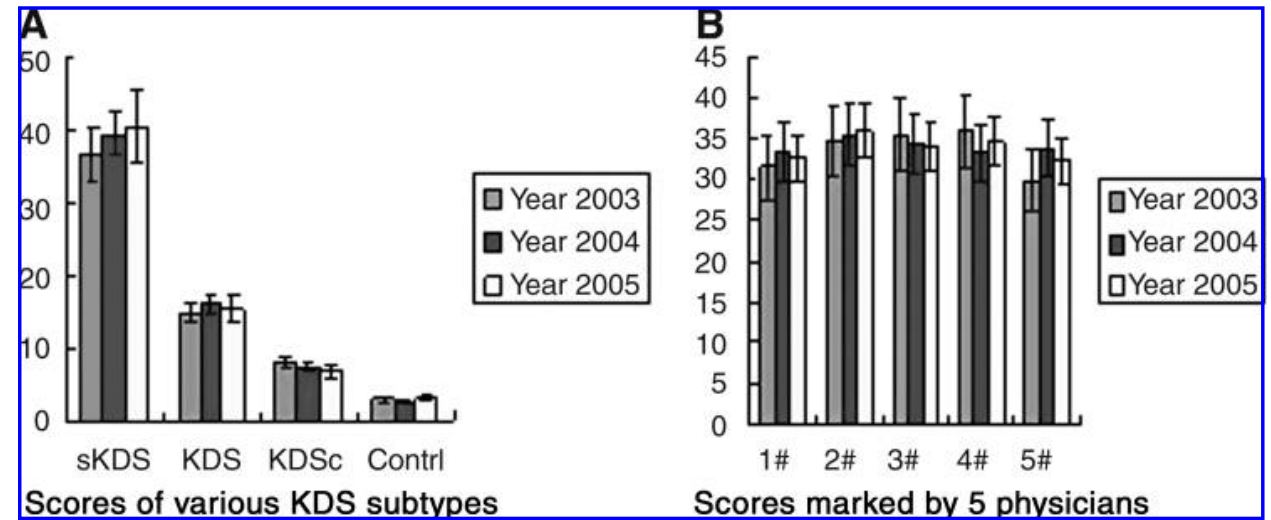

\section{A higher ratio of reappeared genes abnormally expressed among different microchips was observed in the pedigree}

It is interesting that the family data obtained from microarray analysis revealed an extraordinarily high frequency of reappeared genes abnormally expressed among various chips. Reappeared genes (those observed at $\geq 2$ chips as being abnormally expressed) and abnormally expressed genes among various microchips made up $12 \%$ of all differentially expressed genes. For example, two genes, NM_006158 and NM_005252, were all insufficiently expressed in all of the tested chips while two genes, NM_053025 and NM_002619, were all excessively expressed in all chips. This is in contrast to similar microarray studies on non-pedigree KDS patients, ${ }^{11}$ where there were few such reappeared genes observed in different chips. This indicates that pedigree can strongly facilitate KDS research.

\section{Importance of the MAPK pathway in KDS patients}

Pathway analysis showed that MAPK was the most common pathway to be insufficiently expressed in the KDS transcripotome (Table 2). Specifically, there were as many as 15 genes involved in the MAPK pathway observed

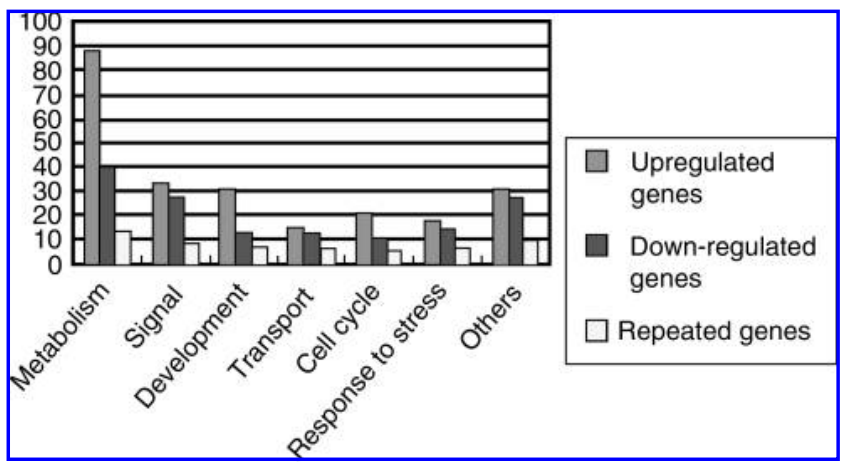

FIG. 3. Functional classification of differentially expressed genes from a Kidney-Yang Deficiency Syndrome (KDS) pedigree. Genes were identified as differentially expressed if the ratios were $\geq 2.0$ or $\leq 0.5$. There were 420 genes/ expressed sequence tags differing significantly in expression level from the control. Of these, 54 genes repeatedly appeared as abnormally expressed genes among different microchips, representing about $12 \%$ of the total.

among the abnormally expressed genes from KDS transcriptomes.

Unexpectedly, genes associated with ATP synthesis pathways were almost all excessively expressed in KDS patients. For instance, genes associated with the oxidative phosphorylation pathway, playing a central role in ATP synthesis, were the most excessively expressed genes in our survey. The up-regulation of oxidative phosphorylation together with glycolysis/gluconeogenesis might act as a kind of feedback mechanism prompted by the low level of energy production in KDS. ${ }^{5}$

We then compared the differentially expressed gene profiles using GSEA. The results show that only subjects with severe KDS demonstrated different gene sets than those of the healthy control group. The MAPK gene set was among those that displayed differences. MAPK is a highly conserved signal-transduction pathway, which plays an important role in the regulation of energy metabolism and thermogenesis. Therefore, the extensive abnormalities of the MAPK pathway may be a factor in the low energy level exhibited by KDS patients (Fig. 4). Pathway analysis suggests that it is not the ATP synthesis system itself that is responsible for the low level of energy output in KDS patient; rather the main monitors, such as MAPK, are to blame.

Table 2. Pathways Related to the Abnormally Expressed Genes of the Kidney-Yang Deficiency SyndRome Pedigree

$P$ Insufficiently expressed pathways

MAPK signaling pathway Amyotrophic lateral sclerosis

Complement and coagulation cascades

Purine and pyrimidine metabolism

Starch and sucrose metabolism

Inositol phosphate metabolism

Neuron diseases

$\mathrm{N}$-glycans biosynthesis

Nucleotide sugars metabolism

Folate biosynthesis

Nicotinate and nicotinamide metabolism
Excessively expressed pathways

Oxidative phosphorylation Purine metabolism Ribosome

Glucose metabolism

Complement and coagulation cascades

Cell adhesion $\mathrm{N}$-glycans biosynthesis Alzheimer's disease Glutathione metabolism Ubiquitin metabolism

Arginine and proline metabolism 


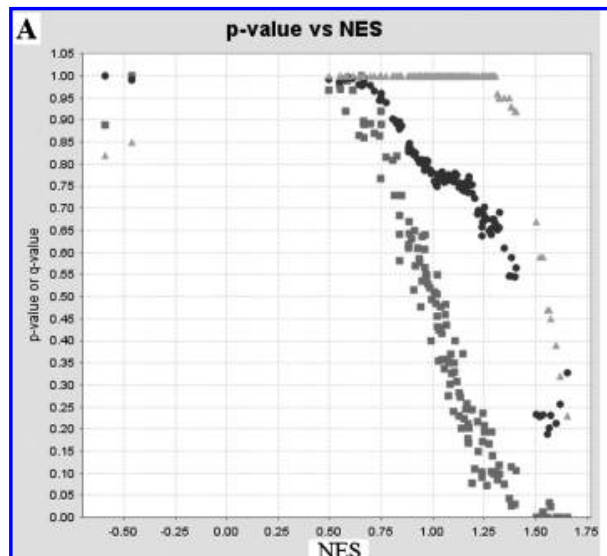

Nominal p-value $D F D R$ q-value FWER p-value

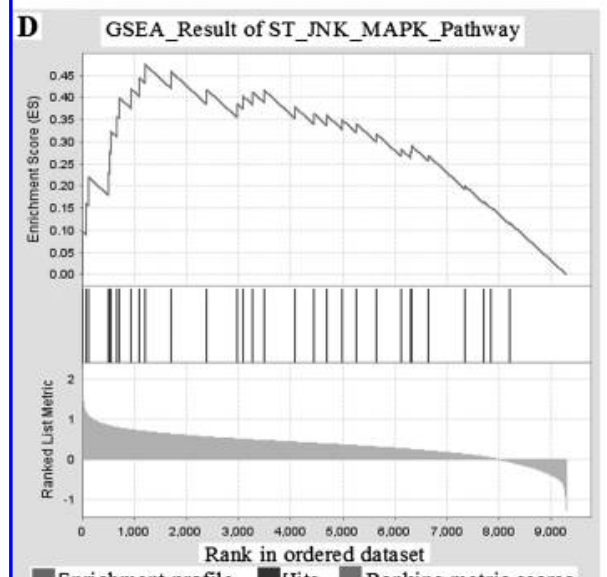

Enrichment profile Hits Ranking metric scores

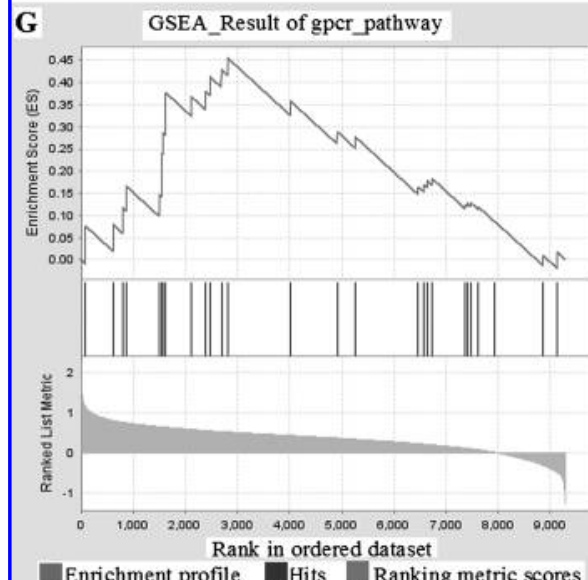

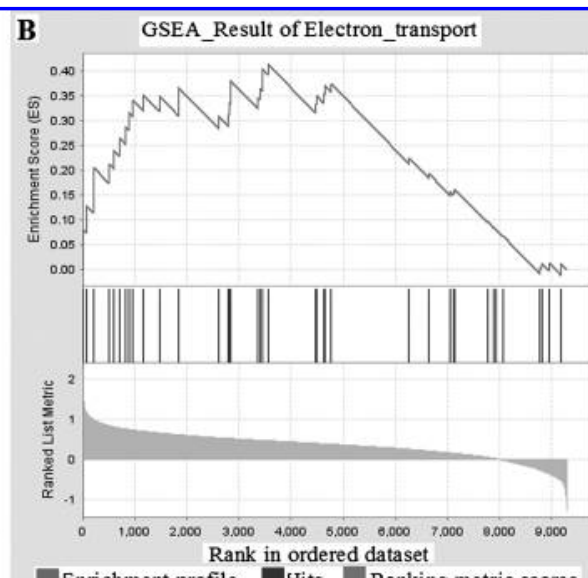

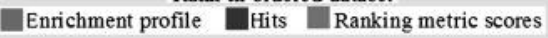

E
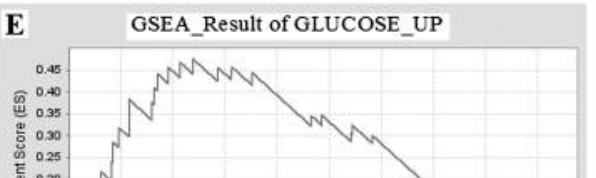

0.25

है. 0.20

0.00.
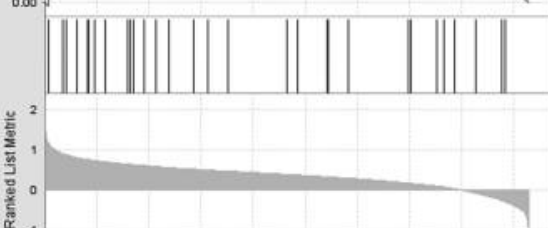

-1

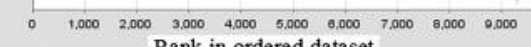

Rank in ordered dataset

Enrichment profile Hits Ranking metric scores

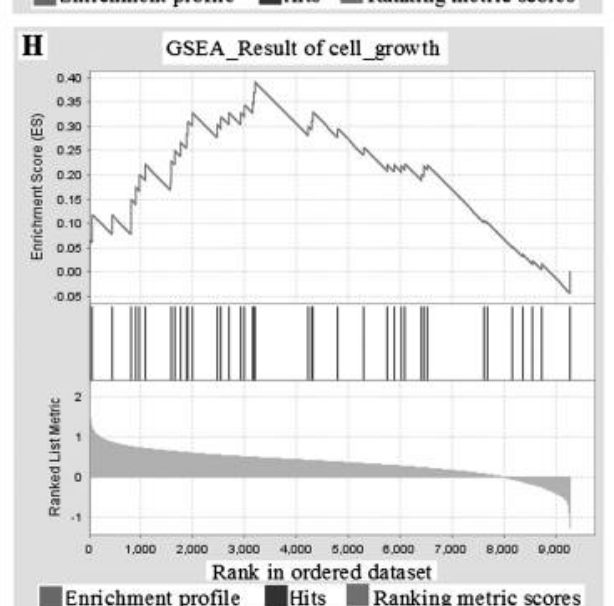

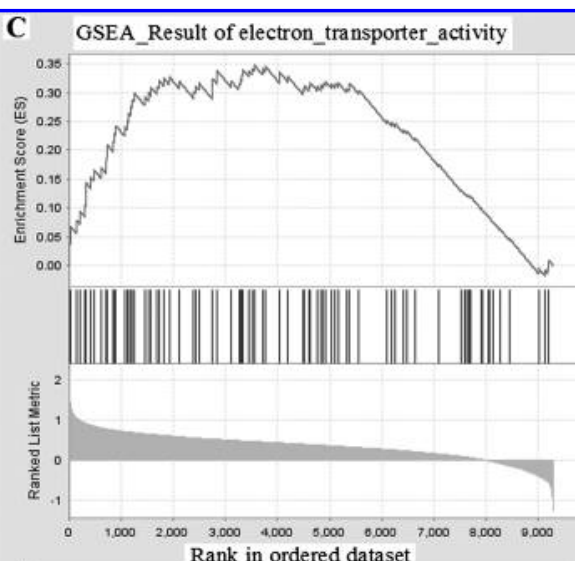

Enrichment profile Hits Ranking metric scores

F GSEA_Result of KRAS_TOP100_KNOCKDOWN

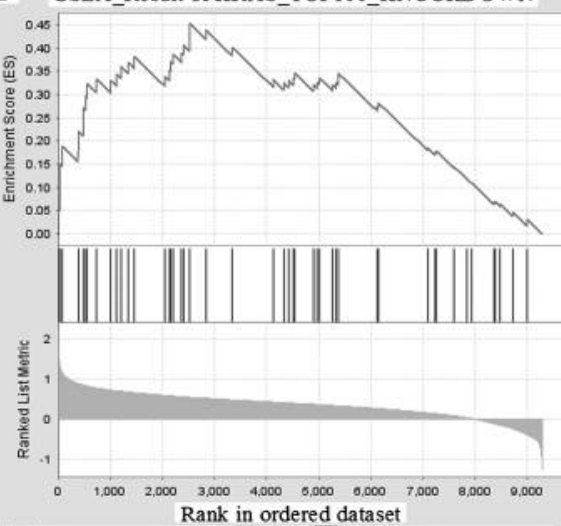

Enrichment profile $\square$ Hits Ranking metric scores

The GSEA result of SCS.

A: $125 / 127$ gene sets had enrichment in phenotype SCS. 12 gene sets are significant at nominal $\mathrm{p}$ value $<5 \%$ and 7 gene sets are significant at FDR $<25 \%$.

B, C, D, E, F, G, H: Profile of the Running ES Score \& Position of Gene Set Members on the Rank Ordered List.

B: electron_transport

C: electron transporter activity

D: ST_JNK_MAPK_Pathway,

E: GLUCOSE UP

F: KRAS TOP100 KNOCKDOWN

G: GPCR pathway

H: Cell_growth_and_or_maintenance.

FIG. 4. The gene set enrichment assay (GSEA) results of patients with severe Kidney-Yang Deficiency Syndrome (KDS) from a KDS family. Eleven patients with severe KDS from a typical KDS family were compared with 10 healthy controls. The criteria for this GSEA analysis was $p<0.05$ and false discovery rate (FDR) $<25 \%$.

\section{Results of RT-PCR and ELISA assays}

RT-PCR results showed that both $\mathrm{ERK}_{2}$ and $\mathrm{MEK}_{1}$ genes were insufficiently represented in KDS patients (Fig. 5). Similarly, results of ELISA assay showed that the activities of $\mathrm{MEK}_{1}$ and $\mathrm{ERK}_{1 / 2}$ of KDS patients were significantly lower than in the control group. This confirms that in the microarray experiments at both the transcript and protein levels, insufficient activity of the MAPK pathway in KDS were ubiquitous.

\section{Discussion}

\section{TCM diagnosis can distinguish KDS subtypes} effectively

Classifying TCM syndromes (Zheng) under the criteria of evidence-based medicine is an important problem. It is characterized by extremely complex elements such as genetics, environment, and disease history. Even though techniques and approaches from multiple disciplines have 


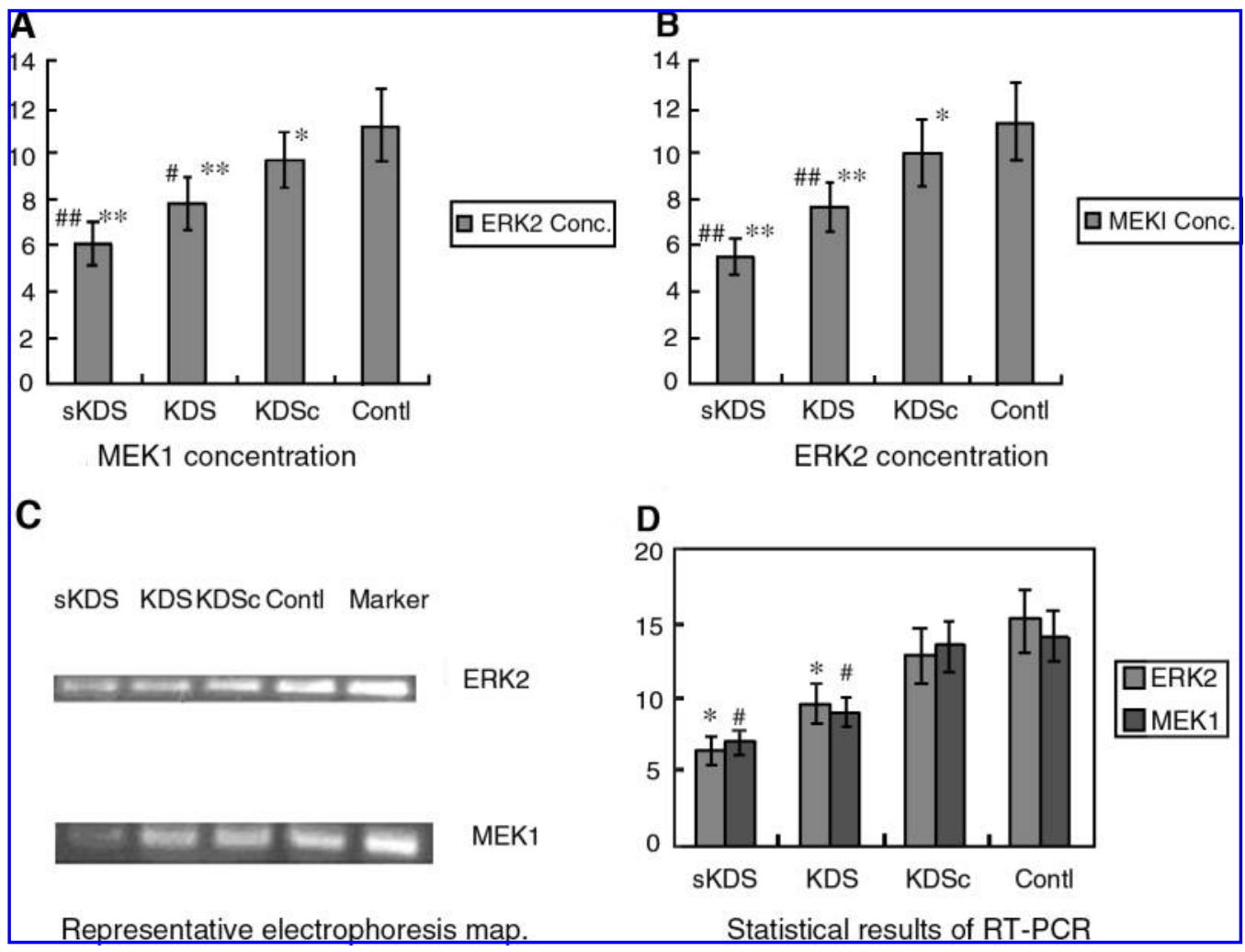

FIG. 5. RT-PCR and ELISA results of a Kidney-Yang Deficiency Syndrome (KDS) family validated the abnormal expressed genes observed in the microarray experiment. (A) The concentrations of phosporylized MEK $_{1}$ assayed by enzyme-linked immunosorbent assay (ELISA) $\left(\mathrm{OD}_{450 \mathrm{~nm}}\right)$ and compared with the healthy control $(p<0.01)$. The relative concentration of phosphorylized amino acid in a special site of the enzyme represents its activity. (B) The concentrations of phosporylized ERK $_{1 / 2}$ assayed by ELISA $\left(\mathrm{OD}_{450 \mathrm{~nm}}\right)$ and compared with the healthy control $(p<0.01)$. (C) Representative electrophoresis map. sKDS, severe KDS; KDSc, KDS constitution; Contl, healthy control. (D) Statistical results (mean \pm standard deviation) of RT-PCR on $\mathrm{ERK}_{2}$ and $\mathrm{MEK}_{1}$, two genes of the MAPK pathway and insufficiently expressed in the microarray assay.

been brought to bear on the subject, classifying TCM syndromes remains a challenge to practitioners of alternative medicine.

We designed our studies based on the principle rules of evidence-based medicine. We used the questionnaire described earlier as a primary screening tool to obtain 82 potential research subjects. Next, we employed 5 experts to identify the KDS patients via a single-blind method. Then, we performed the TCM differentiation three times on the same subjects in order to confirm the validity and reliability of our TCM diagnosis. Finally, we recruited healthy control subjects whose living environment, economic condition, age, and gender ratios were similar to those of the KDS subjects. The results of this research indicate that the time-honored TCM criteria for KDS not only effectively distinguishes KDS patients from healthy people, but also classifies them into various levels of KDS (severe KDS, common KDS, and KDS constitution). The 40-item criteria of KDS diagnosis not only reveals the chronic chill, the major symptom of KDS, but also elicits impaired physiological functions associated with KDS, such as circulation, respiration, digestion, mass and water metabolism, and immunological functions. Therefore, TCM diagnosis for KDS puts chronic chill and low body temperature as revealed by our questionnaire in a much more integrated framework. This serves as the basis for detailed investigation of KDS at a molecular level. Also, the procedures we used for subject recruitment provide us with a substantial number of subjects for the genetic study of KDS.

\section{Pedigree: An important and convenient approach to the study of physical constitution}

The pedigree approach is well accepted among researchers of human genetics. Most disease genes have been directly isolated from pedigrees. Genetics can also be the key factor responsible for myriad TCM syndromes. ${ }^{6}$ Likewise, the pedigree method is useful for further understanding TCM syndromes.

Compared with similar studies published by our group,, 6 we report here on a much more typical KDS family. It has a higher ratio of KDS patients, more family members, and shares more symptoms than those described in previous studies. Furthermore, almost all KDS patients in the family can be effectively treated by You-Gui-Wan, a classical TCM formula for the treatment of $\mathrm{KDS}^{12}$ (data not shown). Our research strongly suggests that inheritance plays a key role in KDS. Therefore, the pedigree approach to researching KDS could be effective in understanding this TCM syndrome.

Microarray results of the KDS family also confirmed our assumption that pedigree is a useful approach for revealing 
the traits of TCM syndromes. For instance, compared to the microarray studies derived from non-pedigree KDS patients, the frequency of reappeared genes was markedly higher among pedigree KDS patients. ${ }^{9}$ This might account for the fact that characteristic genes are heaped up and the expression pattern of certain genes are repeatedly displayed among KDS patients within a family. In our opinion, TCM syndromes may share some features with complex diseases (such as hypertension and diabetes) in their genetic background: all are attributed to multiple genes, show diverse etiopathogenesis, and also develop through various molecular pathways. To this point we have obtained less success in complex diseases than with single gene diseases. However, the pedigree approach is a useful way to study complex diseases. And the pedigree method can also facilitate our further exploration of KDS biomarkers.

\section{KDS transcriptome focuses on energy metabolism}

The results of the microarray tests showed that abnormally expressed genes involved in energy metabolism are significant in the KDS transcriptome and in the pathways related to mass and energy metabolism. Similar results have been observed by others. Shen and colleagues studied the gene networks and signal transduction pathways in a KDS rat model, and found KDS covered the neuroendocrineimmune (NEI) network as a whole-genome impairment. ${ }^{13}$ They discovered significant down-regulation of various neurotransmitters of the hypothalmus-pituitary-adrenalthymus (HPAT) axis, followed by those of the growth and sex hormone related genes. ${ }^{14}$ We also observed many abnormally expressed genes whose products include regulation factors involved in energy metabolism, including a dozen transcription regulators, interleukin 8 (IL-8) mediated inflammatory regulator, and tissue factor pathway inhibitor for blood coagulation.

Although a defined pattern of dysfunction transcriptome has not yet been obtained, we can assume that the marked low level of energy metabolism in KDS may be the leading indication of an overall inbalance of the KDS transcriptome, which gradually intensifies the cold feeling and attenuating physiological functions as a whole. ${ }^{8,12,14}$ Consequently, KDS transcriptome obtained from our experiments revealed that KDS patients suffer from extensive impairments involving large parts of the mass-energy-information carrying network, while the low energy expenditure is merely one of the distinctive features, yet a marked symptom, in KDS patients.

\section{The MAPK pathway in energy metabolism}

The most important discovery presented in this paper might be the universal abnormal expression of certain members of the MAPK pathway. MAPK, one of the critical joint points for many signal transduction pathways (i.e., PKC, RPTK, JAK-STAT), is a key monitor of energy metabolism by regulating the concentration of blood glucose, manipulating the glucose and lipid metabolism, controlling mitochondrial function, and regulating hormonal response for energy output. ${ }^{15-17}$ As described above, abnormally low levels of energy output is the distinguishing symptom of KDS. Hence, in theory, MAPK should suffer some kind of impairment in KDS. Results of pathway analysis indicate the universally abnormal expression of MAPK might be the critical mechanism of insufficient energy output in KDS. The GSEA results confirmed that MAPK is among the signal transduction pathways significantly down-regulated in KDS. Results of both RT-PCR and ELISA assay on MEK $_{1}$ and ERK 2 reconfirmed the fact that some members of the MAPK pathway in KDS patients were insufficiently active.

Shen and colleagues found in a KDS rat model that the NF-kappaB signal transduction pathway exerts a hinge role in the molecule regulating network. ${ }^{13}$ The intensive interaction among mitochondria, MAPK, and NF-kappaB has been reported.$^{16}$ As a pre-inflammation cytokine, NF-kappaB may react more quickly than MAPK in the process of acute modeling of KDS. MAPK, as a predominant hinge and signal transduction highway within all kinds of cells, ${ }^{18-21}$ may play a much more important role in lengthy clinical pathogeneses and TCM syndromes (such as KDS) than does NF-kappaB. Therefore, in our opinion, both MAPK and NF-kappaB are major monitors for energy metabolism, yet they alternatively monitor different TCM syndromes or diverse stages of identical syndromes.

Thermoregulation in human beings and animals is a process with high complexity, integrating a large number of mechanisms and pathways. ${ }^{7,16}$ The extensive insufficient activities of MAPK, and the universal excessive expression of genes involved in ATP synthesis pathways led to our hypothesis that it is not ATP synthesis itself but the critical monitor of energy metabolism such as MAPK that may be responsible for the abnormally low level of energy metabolism in KDS patients.

\section{Acknowledgments}

We are indebted to the National Scientific Fund Committee (NSFC) of China for financial support (30371709 and 30582288). The microarray experiment was performed by the CapitalBio Cooperation, Beijing, China.

\section{Disclosure Statement}

No competing financial interests exist in this article.

\section{References}

1. Marino F. Biotypology. II: Modern concepts. Br Homeopath I 1999;88:178-183.

2. Nagashima K, Yoda T, Yagishita T, et al. Thermal regulation and comfort during a mild-cold exposure in young Japanese women complaining of unusual coldness. I Appl Physiol 2002;92:1029-1035.

3. McQueen MB, Bertram L, Rimm EB, et al. A QTL genome scan of the metabolic syndrome and its component traits. BMC Genet 2003;4:S96.

4. Sood R, Zehnder JL, Druzin ML, Brown PO. Gene expression patterns in human placenta. PNAS 2006;103:5478-5483.

5. Ding WJ, Wang MQ, Xu FY, et al. The study of the temperature and autonomic nerve balance index of a typical cold constitution pedigree [in Chinese]. J Fujian TCM 2003;34:29-32.

6. Pan L, Wang M, Wang JG, et al. Clinical and molecular evaluation of warming and tonic herb treatment for sibling patients of a typical kidney-yang deficiency family. Am J Chin Med 2006;34:387-400.

7. Frank SM, Raja SN, Bulcao CF, Goldstein DS. Relative contribution of core and cutaneous temperatures to thermal comfort and autonomic responses in humans. J Appl Physiol 1999;86:1588-1593. 
8. Wang JG, Pan L, Wu B, Wang M. Familial characteristics of kidney-yang deficiency and cold syndrome. J Toxicol Environ Health A 2006;69:1939-1950.

9. Wu B, Wang M, Wang JG, et al. Establishment of a discriminant mathematical model for diagnosis of deficiency-cold syndrome using gene expression profiling. J Altern Complement Med 2006;12:751-761.

10. Yang L, Wang M, Wu W, Zhang L. Transcriptome analysis of cold syndrome using microarray. Am J Chin Med 2007;35: 609-620.

11. Wang $M Q, W u B$, Feng R, et al. An investigation report on the cold syndrome family [in Chinese]. J Integrat TCM Western Med 2003;12:2385-2386.

12. Li GH, Wang QL, Liu FQ. Experimental study of warming and recuperating Kidney yang by you-gui-yin [in Chinese]. Zhong Xi Yi Jie He Za Zhi 1990;10:547-548, 518.

13. Shen $Z Y$. Studies of gene networks and signal transduction of kidney deficiency syndrome by syndrome differentiation through drug effects [in Chinese]. Zhongguo Zhong Xi Yi Jie He Za Zhi 2005;25:1125-1128.

14. Shen ZY, Huang JH, Chen WH. Comparison of gene expression profiles between rats of Shen deficiency syndrome and KDS differentiated according to therapeutic efficacy of drugs used [in Chinese]. Zhongguo Zhong Xi Yi Jie He Za Zhi 2007;27:135-137.

15. Adler EM, Foley JF, Gough NR, Ray LB. 2007: Signaling breakthroughs of the year. Sci Signal 2008;1:1.

16. Cassarino DS, Halvorsen EM, Swerdlow $\mathrm{RH}$, et al. Interaction among mitochondria, mitogen-activated protein kinases, and nuclear factor-kappaB in cellular models of Parkinson's disease. J Neurochem 2000;74:1384-1388.
17. Pelletier A, Joly E, Prentki M, Coderre L. Adenosine 5'-monophosphate-activated protein kinase and p38 MAPK participate in the stimulation of glucose uptake by dinitrophenol in adult cardiomyocytes. Endocrinology 2005;38: 122-126.

18. Mallon R, Feldberg LR, Kim SC, et al. An enzyme-linked immunosorbent assay for the Raf/MEK1/MAPK signaling cascade. Anal Biochem 2001;294:48-54.

19. Versteeg $H$, Nijhuis $E$, van den Brink GR, et al. A new phosphospecific cell-based ELISA for p42/p44 mitogenactivated protein kinase (MAPK), p38 MAPK, protein kinase $\mathrm{B}$ and cAMP-response-element-binding protein. Biochem J 2000;350:717-722.

20. Su QJ, Chen XW, Chen ZB, Sun SG. Involvement of ERK1/2 and p38 MAPK in up-regulation of 14-3-3 protein induced by hydrogen peroxide preconditioning in PC12 cells. Neurosci Bull 2008;24:244-250.

21. Ge B, Gram H, Di Padova F, et al. MAPKK-independent activation of p38-mediated by TAB1-dependent autophosphorylation of p38 $\alpha$. Science 2002;295:1291-1294.

Address reprint requests to:

Wei Jun Ding, Ph.D.

\#37, Shier Qiao Street

Chengdu 610075

Sichuan Province

China

E-mail: Ding2004wj@yahoo.com.cn 